\title{
Effects of Diet and Physical Activity in an Integrated CVD and T2DM Prevention at High Risk Abdominal Obese Individuals
}

\section{Vera P Simovska*}

The University of St. Kliment Ohridski - Bitola, Republic of Macedonia

\section{Introduction}

Numerous epidemiological, genetically and molecular studies at different population worldwide confirmed that obesity is a significant risk factor for development of main noncommunicable disease (NCD). Abdominal obesity associated with metabolic complications that increase the risk for development of cardiovascular diseases (CVD) and type-2 diabetes (T2DM) is a serious public health problem [1].

Obesity is the complex of a multifactorial phenotype determined of interaction between behavioral factors, mainly energy intake and expenditure that affects body fat mass, genetic variations (hereditary predisposition), different biological factors (sex, vulnerable ages related to increased weight, hormone activity), community situation and environmental factors.

In general, abdominal obesity, CVD and T2DM can be considered as diseases related to unhealthy diet and physical inactivity. Also, it's known that physical activity level (PALs) is an independent predictor of T2DM.

The question of an integrated CVD and T2DM prevention at high risk obese individuals is important scientific problem. Targeted "lifestyle" intervention programme is beneficial especially in abdominal obese individuals. Overall results including our data are additional argument that any other preventive-therapeutic programme is not possible to be applied as alteration for physical activity (PA) [2].

With aim to promote preventive-therapeutic programmes intended for abdominal obese individuals with increased cardiometabolic risk for development of CVD and T2DM, we develop a model of efficient intervention with a proposal - a measure for macronutrient content of diet and increasing cardiorespiratory fitness - $\mathrm{VO}_{2} \max \left(\mathrm{ml}^{-1} \mathrm{~kg}^{-1} \mathrm{~min}^{-1}\right)$.

Within the randomised controlled trial at a group of abdominal obese individuals with presence risk factors (RF) for arteriosclerosis, CVD and T2DM chosen according to a previously given criteria, we suggest the following two types of programmes to be applied:

I. a combined preventive-therapeutic programme composed of moderate hypo-energetic diets with a low atherogenic potential, low glycaemic index (GI) and individually dosed, programmed PA;

II. low fat, low GI, moderate hypo energetic diets.

The programme's objectives are as follows:

1. To examine the influence of the suggested preventivetherapeutic programmes on the basis of a follow up of the intensity of the dynamics and the course of changes of certain anthropometrical variables and indexes for evaluation of the obesity degree and the abdominal distribution of the fat, the functional variables for estimation of the cardiorespiratory fitness and the laboratory variables - indicators for glycoregulation and the metabolism of the lipids;

2. To estimate the risk for diabetes and arteriosclerosis on the basis of determination of the dynamics of changes in the index circumference of the waist/hip ratio (WHR), indexes of the arteriosclerosis: LDL-C/HDL-C, TC/HDL-C, the fasting plasma glucose concentration (FPG), as well as the HbA1c [3], and at the same time estimating the efficiency of the suggested programmes;

3. To propose a method of programming the physical activities on the basis of the common biological suppositions (sex, age, level of aerobic capacities), characteristics of the hypo-kinesis and the obesity degree, in accordance with a suggested preventivetherapeutic diet programme.

\section{Materials and Methods}

In this randomised controlled trial the total number of 82 tested individuals from the adult population, an equal number of men and women were selected. According to the applied type of the therapeutic programme, they were divided into two experimental groups:

$\mathrm{I}^{\text {st }}$-group FAD which applied a combined preventive-therapeutic programme, performed into two phases, out of which the first one was done within a one-month period, $\mathrm{II}^{\text {nd }}$-group $\mathrm{D}$, which applied a diet programme, also in two phases, but as a unique therapeutic procedure, and group $\mathrm{K}$ - a control group of healthy tested individuals.

According to the protocol of work and the originally made health record card, all tested at the programme four groups of changeable variables were followed and calculation indexes were made, as well as examinations of the demographic characteristics.

Analysed: 15 anthropometrics, 13 functional, 12 laboratory and 2 ergonomic variables in order to predict the basic metabolic rate and energetic consumption according to the

After the selection of an adequate preventive-therapeutic programme, the tested individuals are followed within the period of $47.19 \pm 16.22$ days. The results gained regarding the reduction of the weight (g/day) are shown at the initial and final phase of the programme, but also after the $30.05 \pm 6.27$ days.

The research data was processed by application of the software package SPSS of the Kuopio University.

\section{Experimental model}

1. The diet therapy in the first phase of the programme was prepared so that it had a character of "a temporary"

*Corresponding author: Vera P Simovska, Associate Professor, The University St. Kliment Ohridski - Bitola,Coordinator of Postgraduate study for Nutrition, Faculty of Technology and Technical Sciences,Public Health Institutions - Skopje, Krste Misirkov bb, Skopje 1000, Republic of Macedonia, Tel: +38923225402; E-mail: vera.simovska@uklo.edu.mk

Received July 15, 2013; Accepted July 23, 2013; Published July 25, 2013

Citation: Simovska VP (2013) Effects of Diet and Physical Activity in an Integrated CVD and T2DM Prevention at High Risk Abdominal Obese Individuals. J Nutr Disorders Ther 3:e108. doi:10.4172/2161-0509.1000e108

Copyright: () 2013 Simovska VP. This is an open-access article distributed under the terms of the Creative Commons Attribution License, which permits unrestricted use, distribution, and reproduction in any medium, provided the original author and source are credited. 
reduction diet with an energetic value of $1200 \mathrm{kcal}$, a specific macronutrient content $(\mathrm{CH}=48.6 \%, \mathrm{P}=25.7 \%$ and $\mathrm{F}=25.8 \%$ of total DEI), a specific relation among certain fat acids (SFA, MUFA, PUFA), low atherogenic potential under 15 (AHA, EAS), low GI and vitamin-mineral supplementation. The second phase of the programme was the increased energetic value of the diet therapy for $200 \mathrm{kcal}$ per day [4].

2. The PA programme, with individually dosed loading intensity which is $>50 \%$ Heart rate maximum reserve (\% HRmax reserve) in the first phase and $>60 \%$ HRMax reserve in the second phase was prepared on the basis of the initial level of cardiorespiratory fitness - $\mathrm{VO}_{2} \max \left(\mathrm{ml}^{-1} \mathrm{~kg}^{-1} \mathrm{~min}^{-1}\right)$ of the obese individuals [4].

\section{Method for programming PA}

A. Basic criteria for distribution of the tested patients into four programme's groups of different physical activity levels ("PALs") is in accordance to the initial level of cardiorespiratory fitness, expressed into metabolic equivalents (METs). METs is calculated by the equation on $\mathrm{VO}_{2} \max \left(\mathrm{ml}^{-1} \mathrm{~kg}^{-1} \mathrm{~min}^{-1}\right) / 3,5$.

B. The choice of the type of the PA is made in relation to the energetic cost, expressed in METs, which is in accordance to the initial level of the aerobic capacities of each tested individual.

C. The level of intensity in the appropriate "PALs" groups is $50 \%$ of the initial level of cardio-respiratory fitness. Training pulse (\% HRmax reserve) is calculated using equation by Karvonen Martin.

D. The suggested method is based on the WHO classification of the cardiorespiratory fitness (1973).

An estimation of the efficiency of the preventive-therapeutic programmes could be made by following the dynamics of changes of certain indicators of the situation of the glycoregulation and the metabolism of the lipids, as well as by following certain anthropometrical and functional indicators of the cardio-respiratory fitness of the obese individuals.

A criterion for estimation of the efficiency of the programmed PA is the significantly higher reduction of the tested risk factors and the risk for arteriosclerosis and T2DM associated with abdominal obesity.

We suggested four groups to be formed through modelling of the programme of the physical activity whose energetic value corresponds to the individual abilities of each individual tested. Programmed physical activities should be applied $30 \mathrm{~min} / 5-6$ times a week, or 60 $\mathrm{min} / 3$ times a week.

The aim of the selected physical activities though the modelling of this programme is to encourage specific biological changes, which are connected with the increased cardiorespiratory fitness in individuals with abdominal obesity and RF for arteriosclerosis, CVD and T2DM.

At the same time, estimation was made for:

1) The morphologic status and the structural components;

2) Functional capabilities of the cardiorespiratory system;

3) The metabolism of the lipids and the glycoregulation;

4) Indexes for arteriosclerosis;

Both experimental and control groups have been included in the statistical analysis and examined in the initial and final phase.
In order to determine the dynamic changes of certain variables during the application of the programmes that functions from the initial to the final time phase, we made an analysis, observing the time changes as well. Except for this conclusion in terms of time changes, the direction of changes in both experimental groups is analysed, upon which the behaviour between the two groups is determined. We put into three parts the dynamic changes of all variables and indexes observed, as well as those analysed according to the type of the applied preventive-therapeutic programme, according to which the intensity and the direction of changes are determined.

\section{Results}

Significantly more intensive dynamic changes are determined in the following variables and indexes in the FAD group, comparing it with group D (Figure 1):

- Higher reduction of body weight in the first phase and the whole period of the programme;

- Higher reduction of the total body fat $(\% \mathrm{~F})$, the lean body mass in physiological range (LBM $\mathrm{kg}$ ) and abdominal accumulated fat tissue (WHR);

- Decrease of the arteriosclerosis risk (LDL-C/HDL-C and TC/ HDL-C);

- Increase of the level of the HDL-C in comparison with the $\mathrm{D}$ group, in which a significant decrease regarding the initial values appeared;

- Increase of the cardiorespiratory fitness which is $17.16 \%$ in relation to the initial values;

- Decrease of the number of tested individuals with under average aerobic abilities for $30 \%$, out of which in the final phase of the programme $80 \%$ of the tested in the FAD group had normal and above average abilities of the aerobic capacity;

- The tested had a significantly lower average value of the systolic blood pressure in still position in the FAD group, compared to the $\mathrm{D}$ group

- Significantly lower average value of the fasting plasma glucose concentration (FPG), as well as in the HbAlc.

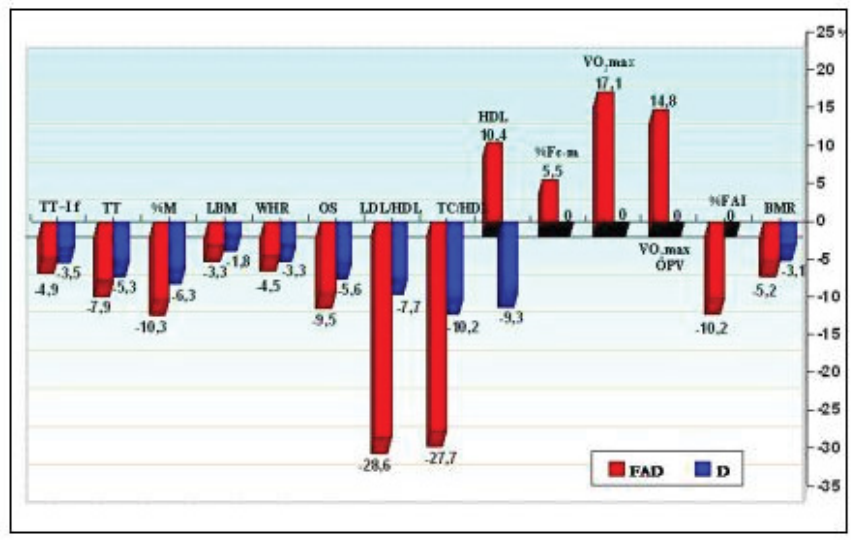

Figure 1: Significant changes in the level of VO max and "major" RF for CVD and T2DM between FAD (physical activity and diet) and D (diet) groups. 
Citation: Simovska VP (2013) Effects of Diet and Physical Activity in an Integrated CVD and T2DM Prevention at High Risk Abdominal Obese Individuals. J Nutr Disorders Ther 3:e108. doi:10.4172/2161-0509.1000e108

\section{Conclusion}

Research results confirm a significant therapeutic efficiency of the recommended PA program in combination with a suitable diet therapy prescribe the possibilities for prevention and therapy of:

1. Obesity associated with expression of certain RF for arteriosclerosis and the consequential development of the process of arteriosclerosis;

2. Metabolic complications combined with insulin resistance that increase the cardiometabolic risk of the development of CVD and T2DM at people with increased abdominal fat distribution.

\section{References}

1. Pekka P, Pirjo P, Ulla U (2002) Influencing public nutrition for non-communicable disease prevention: from community intervention to national programme-experiences from Finland. Public Health Nutr 5: 245-251.

2. Simovska V (2008) Effects of diet therapy and programmed physical activity on obesity and its complications. Ed. Ministry of Science and Education, Republic of Macedonia, Menora, Skopje.

3. Alberti KG, Zimmet P, Shaw J (2007) International Diabetes Federation: a consensus on Type 2 diabetes prevention. Diabet Med 24: 451-463.

4. Simovska-Jarevska V, Vidin-Jarevska M (2008) A proposal-a measure in the modern concept of type 2 diabetes prevention focused on macronutrient content of diet and increasing cardio respiratory fitness at high-risk obese subjects. $5^{\text {th }}$ World Congress on Prevention of Diabetes and its Complications (WCPD 2008), Helsinki, Finland. 